in this disorder. A gap in the literature concerning early stages of the disorder is identified.

Conclusions: The observed emotional features of BPD have important negative consequences for daily life functioning. Studies of social cognition in BPD can help to better identify the key factors underpinning these emotional disturbances in BPD. More research is needed, however, in the early stages of the disorder, prior to the effects of chronic stress and recurrent mental state pathology associated with adult forms of BPD, to better evaluate the primacy and core nature of social cognition impairments in BPD.

06-03

\section{What is the evidence that social cognition deficits in schizophrenia represent a vulnerability marker?}

\section{Loughland}

Neuroscience Institute of Schizophrenia and Allied Disorders (NISAD), C/-Centre for Mental Health Studies, Newcastle, Australia

Background: Patients with schizophrenia are observed to have marked deficits across several social cognition domains including theory of mind (TOM), social perception and emotion processing. These deficits are observed at illness onset (TOM, Polowsky et al. 2002) and across illness phase (TOM, Janssen et al. 2003), and differ in presentation from those observed in other diagnostic groups (eg depression, Garety \& Freeman 1999; affective disorder, Loughland et al. 2002), suggesting they may represent a trait marker for the disorder. The aim of this paper was to review current findings with regard to social cognition deficits in schizophrenia and to examine the evidence that these deficits may represent a vulnerability marker.

Method: A narrative review of the research literature was undertaken and supplemented with the current research findings of the author that investigate emotionprocessing deficits in people with schizophrenia, first-degree relatives, affective disorder and healthy non-psychiatric controls.

Results: Findings from TOM, social context and emotion processing provide evidence that healthy firstdegree relatives and other at-risk groups show similar, but less severe, social cognition deficits to those observed in schizophrenia (Cornblatt \& Keilp 1994; Frenkel et al. 1995; Toomey et al. 1999; Loughland et al. 2004).

Conclusions: The results support the notion of a social cognition vulnerability marker for schizophrenia disorder. Differential patterns of performance on social cognition tasks may help detect those potentially at risk of developing schizophrenia and provide a potential method for developing endophenotypes for examining diagnostic, genetic and therapeutic issues in schizophrenia.

06-04

\section{Social cognition in nonforensic psychopathy: further evidence for a dissociation between intact 'theory of mind' and impaired emotion processing}

\author{
M Aylett', M Mahmut', R Langdon ${ }^{2,3,4}$, M Green ${ }^{2,3,5}$ \\ 1Psychology Department, Macquarie University; ${ }^{2}$ Macquarie Centre for Cognitive \\ Science (MACCS), Macquarie University; ${ }^{3}$ Neuroscience Institute of Schizophrenia \\ and Allied Disorders (NISAD), New South Wales, Australia; ${ }^{4}$ Schizophrenia \\ Research Unit, Sydney South West Area Health Service; and \\ Enniversity of New South Wales, Sydney, New South Wales, Australia
}

Background: Psychopathy is a developmental disorder characterized by antisocial behaviour identified in forensic settings using the Psychopathy Checklist-Revised (PCL-R; Hare 1991). Forensic psychopaths, like autistic individuals, show social cognition abnormalities linked to amygdala dysfunction (eg impaired recognition of negative affect). These developmental disorders differ, however, with respect to theory of mind (ToM); while psychopaths are adept at imputing others' causal mental states (intentions/beliefs), ToM deficits characterize autism. In line with recent research that has used nonforensic groups to further examine the dissociation of sociocognitive skills in psychopathy, this study investigated conscious and preconscious processing of facial affect, ToM and empathy in a university sample assessed for psychopathic traits using the Self Report Psychopathy Scale III (SRP-III; Paulhus et al. in press), an instrument styled on the PCL-R.

Method: About 416 university students completed the SRP-III. Sixty individuals with high ( $>75$ percentile), medium (40-60 percentile) and low ( $<25$ percentile) psychopathic tendencies (20 per group) were then called back for experimental testing. Tasks assessed facial affect recognition, affective priming (using subliminally presented facial expressions) and ToM. The Emotional Empathy Questionnaire (Mehrabian \& Epstein 1972) was also administered.

Results: While groups did not differ in their ToM abilities, psychopathic tendencies were associated with poor recognition of negative affect (eg disgust), an absence of affective priming and reduced empathy.

Conclusions: Nonforensic and forensic psychopaths appear alike; these individuals know how others 
think but neither know (explicitly or implicitly) nor care how others feel. The role of the amygdala in the development of ToM in autism and psychopathy is discussed.

06-05

\section{Theory of mind in Williams syndrome assessed using a nonverbal task}

\section{Porter}

Macquarie Centre for Cognitive Science (MACCS), Macquarie University, Sydney, Australia

Background: Williams syndrome (WS) is a rare genetic disorder associated with intellectual impairment, good verbal skills and an unusual social personality. Despite extensive interest in the area, it remains unclear whether theory of mind (ToM) abilities are impaired or intact in WS. This uncertainty most likely relates to the wide range of measures used to assess ToM abilities and the unique profile of strengths and weaknesses associated with the syndrome. The aim of this study was to examine ToM in WS using a nonverbal picture sequencing task. The picture sequencing task was selected so that the WS individuals could not rely on their good verbal skills when performing the task. The paper also aimed to further explore heterogeneity within WS and the possibility of WS subtypes.

Methods: Langdon et al.'s (1997) picture sequencing task was administered to 30 individuals with WS and to normal chronological-age-matched and mentalage-matched controls. The picture sequencing task assesses understanding of pretence, intention and false belief, while controlling for social script knowledge and physical cause-and-effect reasoning.

Results: Results indicated a specific deficit in understanding of false belief within the WS group. There was also evidence of heterogeneity in the WS group, with the false belief impairment restricted to only a particular subgroup of WS individuals identified originally by Porter and Coltheart (2005).

Conclusions: Our research, using a nonverbal task to assess ToM, indicates impaired false belief understanding in a select group of WS individuals. There are some indications that the deficits in social understanding in this group go beyond an impaired ToM.

\section{Brain Stimulation Approaches in the Treatment of Depression}

\section{Loo}

School of Psychiatry, University of New South Wales, Sydney, New South Wales, Australia

\section{Overview}

This symposium will discuss current clinical and potential future brain stimulation techniques for the treatment of depression: electroconvulsive therapy (A/Professor Loo), transcranial magnetic stimulation (Professor Mitchell), vagus nerve stimulation (Doctor Trollor), transcranial direct current stimulation, deep brain stimulation and parallels with psychosurgery (Doctor Malhi). Each talk will present an overview of the current state of the field, recent original research and possible mechanisms of action. The session will conclude with a panel discussion on similarities and differences in the neurobiological effects of these different brain stimulation approaches.

07-01

\section{ECT - future directions: a trial of the effectiveness and safety of four forms of ECT, bitemporal, bifrontal, right unilateral and right unilateral (ultrabrief pulsewidth)}

\author{
C Loo', P Sheehan², B Lyndon² \\ 'School of Psychiatry, University of New South Wales, Sydney, Australia; and \\ ${ }^{2}$ The Northside Clinic, Sydney, New South Wales, Australia
}

Background: Electroconvulsive therapy (ECT) is a highly effective treatment for depression, but its use is limited by the risk of associated cognitive side-effects. This study aimed to investigate the relative effectiveness and cognitive side-effects of ECT given in four different forms, varying in electrode placement and pulsewidth, to determine the optimal form of ECT.

Methods: Depressed in-patients referred for ECT in a private clinic in Sydney were recruited after giving informed consent. Subjects received the form of ECT prescribed by their treating psychiatrist [bitemporal at 1.5 times seizure threshold (1.5 ST), bifrontal at $1.5 \mathrm{ST}$, right unilateral at $5 \mathrm{ST}$ or right unilateral with ultrabrief pulsewidth at $6 \mathrm{ST}$ ]. Mood (MADRS) and neuropsychological functioning (digit span, Rey Auditory Verbal Learning Test, Rey figure, word 